\title{
Effect of photobiomodulation in secondary intention gingival wound healing - a systematic review and meta-analysis
}

\author{
Pooya Ebrahimi ${ }^{1}$, Mahdi Hadilou' ${ }^{1}$, Ferdos Naserneysari ${ }^{1}$, Amirmohammad Dolatabadi ${ }^{1}$, Rana Tarzemany ${ }^{2}$, \\ Nafiseh Vahed ${ }^{3,4}$, Leila Nikniaz ${ }^{5}$, Reza Fekrazad ${ }^{6}$ and Leila Gholami ${ }^{7 *}$
}

\begin{abstract}
Background: Photobiomodulation is widely being used to improve the wound healing process in dentistry and a vast majority of studies have proven its benefits. But there are plenty of knowledge gaps according to the optimal laser characteristics which should be used to maximize the healing effects of lasers. The goal of this systematic review and meta-analysis was to determine the effect of photobiomodulation (PBM) as an adjunctive treatment to periodontal therapies to evaluate secondary intention gingival wound healing and post-operative pain.
\end{abstract}

Methods: Five databases (PubMed, Embase, Scopus, ProQuest, and Web of Sciences) were searched up to November 30,2020 , for clinical trials that reported the result of the application of PBM on secondary gingival healing wounds and post-operative pain and discomfort after periodontal surgeries. Two independent reviewers selected the eligible studies and the outcomes of interest were extracted. The quality of eligible studies was assessed using the Cochrane Handbook for Systematic Reviews of Interventions.

Results: Ultimately, twelve studies were included in this review. The application of PBM as an adjunct to periodontal surgeries resulted in a significant improvement in wound healing indices. The Landry wound healing index at the 7 th post-operative day was significantly improved $(\mathrm{SMD}=1.044[95 \% \mathrm{Cl} 0.62-1.46] ; \mathrm{p}<0.01)$ in PBM + surgery groups compared to the control groups. There was also a statistically significant increase in the complete wound epithelialization $(R R=3.23[95 \% \mathrm{Cl} 1.66-6.31] ; \mathrm{p}<0.01)$ at the 14th post-operative day compared to the control groups. The methods used to assess the post-operative pain were heterogeneous, and therefore the results were limited which made the meta-analysis for post-operative pain assessment not possible.

Conclusion: Based on the results of this review, PBM can be effectively used as a method to improve secondary intention wound healing. High-quality randomized clinical trials, however, are needed in the future to identify the optimal PBM irradiation parameters and the effect of PBM on post-operative pain.

Keywords: Photobiomodulation (PBM), Low-level laser therapy (LLLT), Secondary intention wound healing, Periodontal surgery

\footnotetext{
*Correspondence: I.gholami@hotmail.com

${ }^{7}$ Department of Periodontics, Dental Research Center, School

of Dentistry, Hamadan University of Medical Sciences, Shahid Fahmideh

Blvd, 654178-38741 Hamadan, Iran

Full list of author information is available at the end of the article
}

\section{Background}

Improvement of wound healing after periodontal surgeries is a critical factor in achieving favorable clinical results [1]. Optimal wound healing and reduction in the severity and duration of post-operative pain result in a better prognosis and outcome of the periodontal treatment and 
patient satisfaction [2]. Post-operative discomfort or pain is a subjective experience, and the process of wound healing is multifactorial $[3,4]$. This pain is sometimes associated with a delayed wound healing. It is influenced by several emotional, clinical, and iatrogenic causes including stress and psychological condition, patient's earlier experiences, type and duration of surgery, surgeon's experience and skills, and also the type of wound closure (primary or secondary) [5-8]. Some of the common medications and methods used by clinicians to improve wound healing after periodontal surgeries include the application of chlorhexidine with or without alcohol [9, 10], nutritional supplementations [11], and antibiotics such as azithromycin [12], vitamin D [13], professional tooth cleaning [14], and the use of fibrin sealants instead of sutures [15].

Secondary intention healing wounds can be associated with considerable discomfort and delayed healing compared to primary intention healing wounds after the periodontal flap surgeries. Gingivectomies, depigmentation procedures, and harvesting free gingival graft tissues from the palatal area are common secondary intention healing wounds. This healing type occurs when the wound site is left open to heal mostly by granulation, contraction, and epithelialization. Moreover, we encounter more scar formations and contraction [8].

The application of photobiomodulation (PBM) as an adjunctive therapy to improve wound healing has attracted the attention of many researchers in recent years $[16,17]$. PBM, includes the application of laser or light-emitting diode (LED) beams for stimulation of healing, relieving pain, and reducing inflammation [18]. Numerous studies have shown the positive inductive effects of photobiomodulation on the viability and proliferation of skin and gingival fibroblast cells, in vitro [16, $17,19,20]$. Therefore, this biophysical approach has been considered as a treatment modality which can stimulate the endogenous healing process. The main mechanisms considered for the observed biological response is the absorbance of low-level light irradiation by cellular photoreceptors or ROS production and subsequent generation of highly reactive, transient biochemical intermediates, changes in cellular ionic gradients or cell polarity and ultimate increase in ATP production, recruitment of transcription factors and increase in cell activity. This results a secondary phase of responses including cell proliferation, differentiation and migration, angiogenesis, production of growth factors and matrix synthesis which contribute to promotion of wound healing [21-24].

Clinically, PBM has also been reported to result in a decreased pain sensation, enhancement of keratinization [25-27], and improvements in periodontal clinical characteristics such as enhancement in clinical attachment level (CAL) and probing depth (PD) [28, 29]. Several studies have emphasized the significant effect of PBM on post-operative pain reduction and wound healing improvement after periodontal surgeries, although there exist some controversies in the reported results $[20$, 30-34].

The characteristics of PBM irradiation parameters need to be considered as an important factor in order to achieve an optimal dose of irradiation, as a small amount or too high irradiation dose could have no effect or undesirable inhibitory results on wound healing outcomes [35]. Various laser wave lengths and settings have been used to promote oral wound healing and there is a large amount of information about PBM application in the field of wound healing. However, the effects on open oral soft tissue wounds and the most fitting laser characteristics to improve the healing of these types of wounds have not been specified to date. Therefore, the present review, aimed to determine the effectiveness of the application of $\mathrm{PBM}$ as an adjunctive treatment in periodontal surgeries to improve secondary intention wound healing and postoperative pain and find an evidence-based answer to this question:

\section{"Does the application of PBM as an adjunct, improve the secondary intention wound healing after periodontal soft tissue surgeries?"}

\section{Methods}

\section{Protocol registration}

All study concepts and details were recorded and published in the international prospective register of systematic reviews (PROSPERO). (Registration ID: CRD42020192403).

\section{Focused question}

The Preferred Reporting Items for Systematic Review and Meta-Analysis (PRISMA) [36] guidelines were respected.

The addressed PICO was: "Can photobiomodulation improve soft tissue secondary wound healing and postoperative pain after periodontal surgeries?".

\section{Selection criteria}

The eligibility criteria for studies to be included in this review were based on the following PICOS:

(Population): the participants who had undergone periodontal, soft tissue surgeries resulting in a secondary intention healing wound (depigmentation, gingivectomy, or free gingival soft tissue grafts) and without any systemic conditions.

(Interventions): the intervention groups that were treated with adjunctive PBM (Laser or LED) irradiation on the gingival wound site after the surgery. 
(Outcomes): our outcome measures of interest were wound healing parameters such as Landry Wound Healing Indices (WHI), epithelization, and pain after surgery.

(Study design): this review was restricted to controlled trials published in English.

All animal studies, opinion articles, in vitro studies, reviews, unpublished studies, abstracts, and articles in which the patients had systemic disease, the wounds were sutured, and interventions including flap elevation were excluded.

\section{Search strategy}

The authors (NV and $\mathrm{MH}$ ) performed an extensive search in the online databases of Embase, PubMed, Scopus, ProQuest, and Web of Science in search of relevant studies which had been published before 30 November 2020 . The literature search was conducted using the modified type or combination of the following words: "photobiomodulation", "PBM", "low level laser therapy", "low intensity laser therapy", "LLLT", "low level light therapy", "low power laser therapy", "low power laser irradiation", "periodontal surgery", "wound healing", "gingivectomy", "pigmentation", "depigmentation", "palatal donor site" (Additional file 1: Appendix 1). The reference lists of included articles were also manually searched. Gray literature search of evidence was also conducted.

\section{Screening methods and data abstraction}

Two reviewers (AD and FN) independently screened the studies in three steps. The first step was the removal of duplicates. After assessing the remaining studies based upon the titles and abstracts, then, ruling out the irrelevant, the authors reviewed the full texts of selected articles. Full texts were included, considering the eligibility criteria. If there were opposing opinions among the reviewers, they were referred to a third reviewer (LG), then the final decision was made through a group discussion.

Data were extracted from the full text of selected studies for the following factors: author/year, study type, the number of subjects, type and site of the procedure, study groups, evaluated criteria and study outcome, use of analgesics, and follow-ups.

Considering the importance of irradiation parameters in PBM therapies, in another table, the following data concerning the irradiation parameters applied were extracted:

Laser type, wavelength, application mode, output power, total exposure time, total energy, beam diameter or probe spot size, energy density, distance to the intervention site, method of application, frequency of laser treatment, or the number of irradiation sessions.

\section{Risk of bias among the studies}

Evaluation of the risk of bias among the included articles was performed associated with the following concepts by the reviewers:

Random sequence generation, allocation concealment, blinding of participants and personnel, blinding of outcome assessment, incomplete outcome data, selective reporting, and other bias.

To assess the risk of bias in each study, the Cochrane Handbook for Systematic Reviews of Interventions was used [37]. Both of the assessors discussed and resolved any disagreements.

\section{Data synthesis}

The meta-analysis was done using Comprehensive Meta-Analysis (CMA) software version 2. The relative risk (RR) and mean differences (MD) were used for dichotomous and continuous data, respectively, considering a 95\% confidence interval. To demonstrate the achieved results, forest plots were utilized. The statistical heterogeneity was recognized by the application of the chi-square test and $\mathrm{I}^{2}$ value [38]. To check publication bias and illustrate it as a funnel plot, Egger's test was done [39].

\section{Results}

\section{Study selection}

After the initial search, 3076 studies were found. The authors removed the duplicates $(\mathrm{n}=789)$ and evaluated the titles and the abstracts. A number of 2269 articles were found to be not relevant to the study's objective and were excluded. Twenty studies were selected for a thorough evaluation of full-texts in which, eight studies were put aside as they did not meet the eligibility criteria (Additional file 2: Appendix 2). Finally, twelve studies were selected as are shown in the study selection flow diagram (Fig. 1) [17, 19, 20, 30, 32-34, 40-44].

\section{Characteristics of included studies}

Table 1 shows the characteristics of the included studies. All of the twelve included studies were clinical trials. Trials were originated from Brazil [30, 32, 33, 40], Iran [19], Turkey [17, 34, 41, 43], and India [20, 42, 44].

The type of procedure in four of the included studies was gingivectomy [20, 30, 41, 42], it was gingival grafting in five studies $[17,19,34,40,43]$, gingivoplasty in two studies [32, 33], and one study used surgical stripping for gingival hyperpigmentation [44]. The overall number of participants among the studies ranged between 10 and 40. In all studies, smoking history was absent, and 


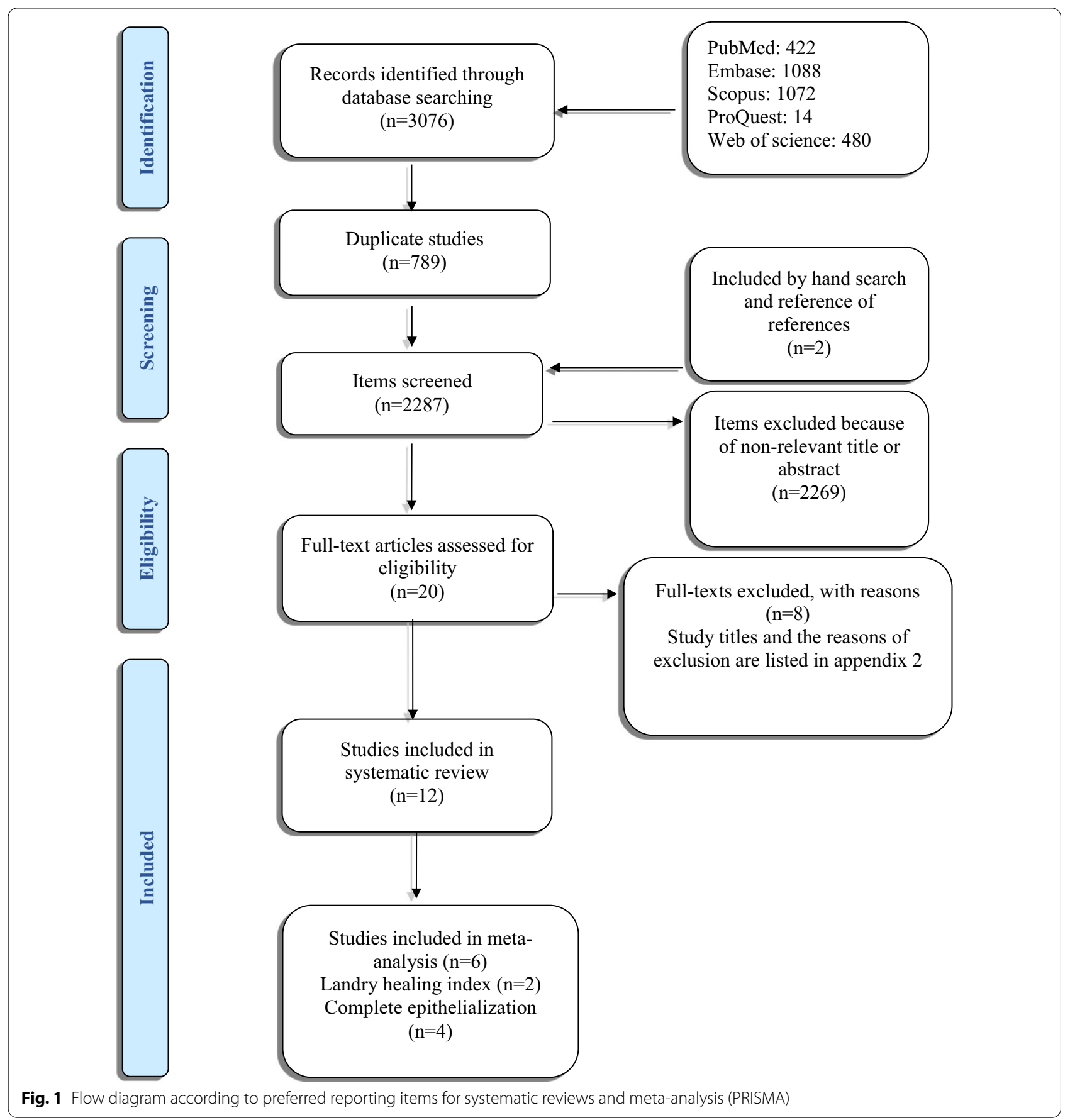

the follow-up frequencies were between 2 and 13 times (Table 1).

\section{Assessment of risk of bias among the studies}

The summary of the risk of bias is shown in Fig. 2. Seven articles were found to have issues regarding randomization or concealment of allocation $[20,30,32,33$,
$40,41,44]$ (selection bias). The main cause of bias in the included studies was related to blinding. Five items did not blind the participants or the personnel [17, 32, $33,40,41]$, and it was unclear in two studies [20,30] (performance bias). Also, two studies did not blind the outcome assessor $[20,41]$ and it was unclear in one study [34] (detection bias). Also, four studies had a bias in reporting $[17,19,33,43]$ (attrition or reporting bias). 


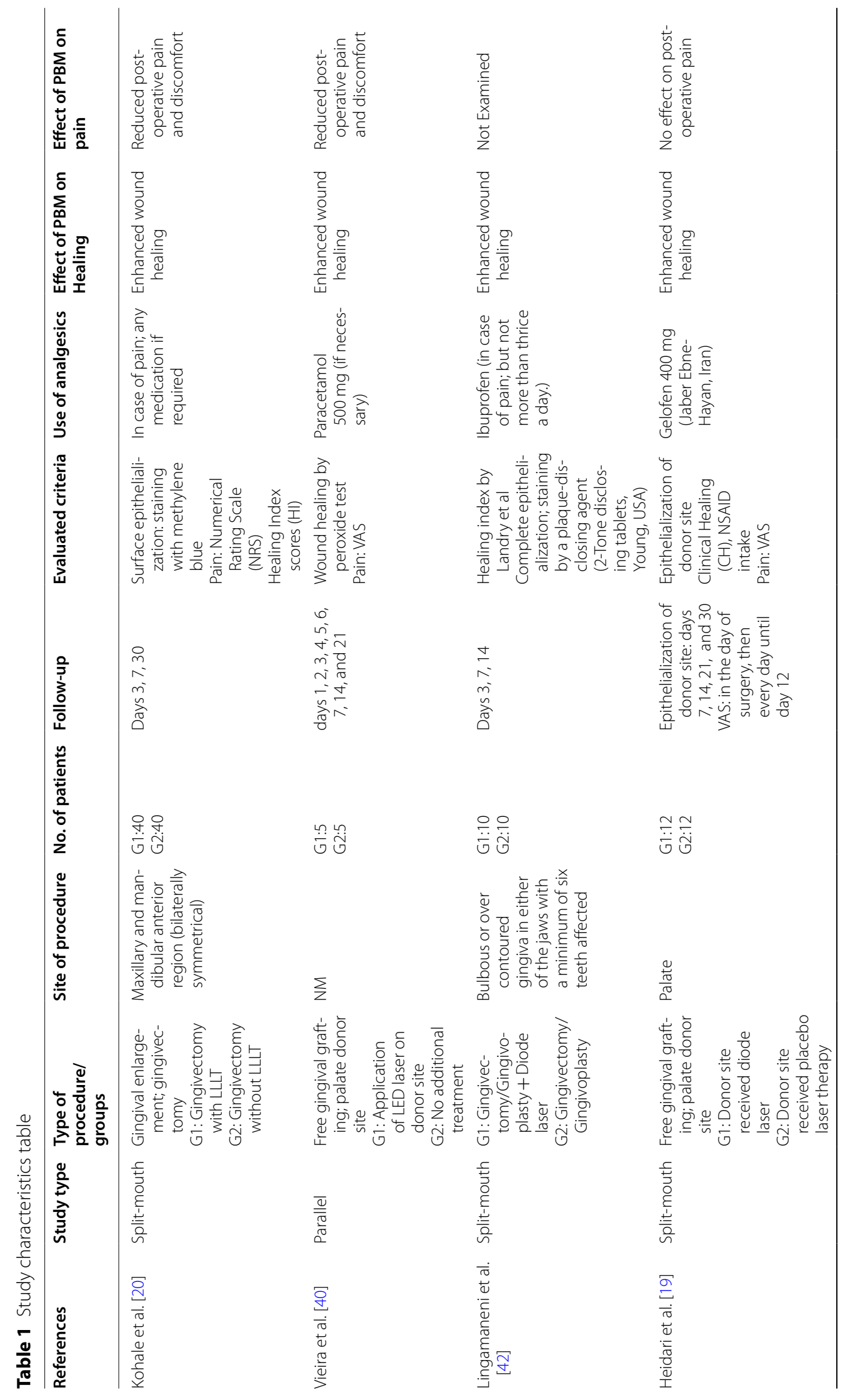




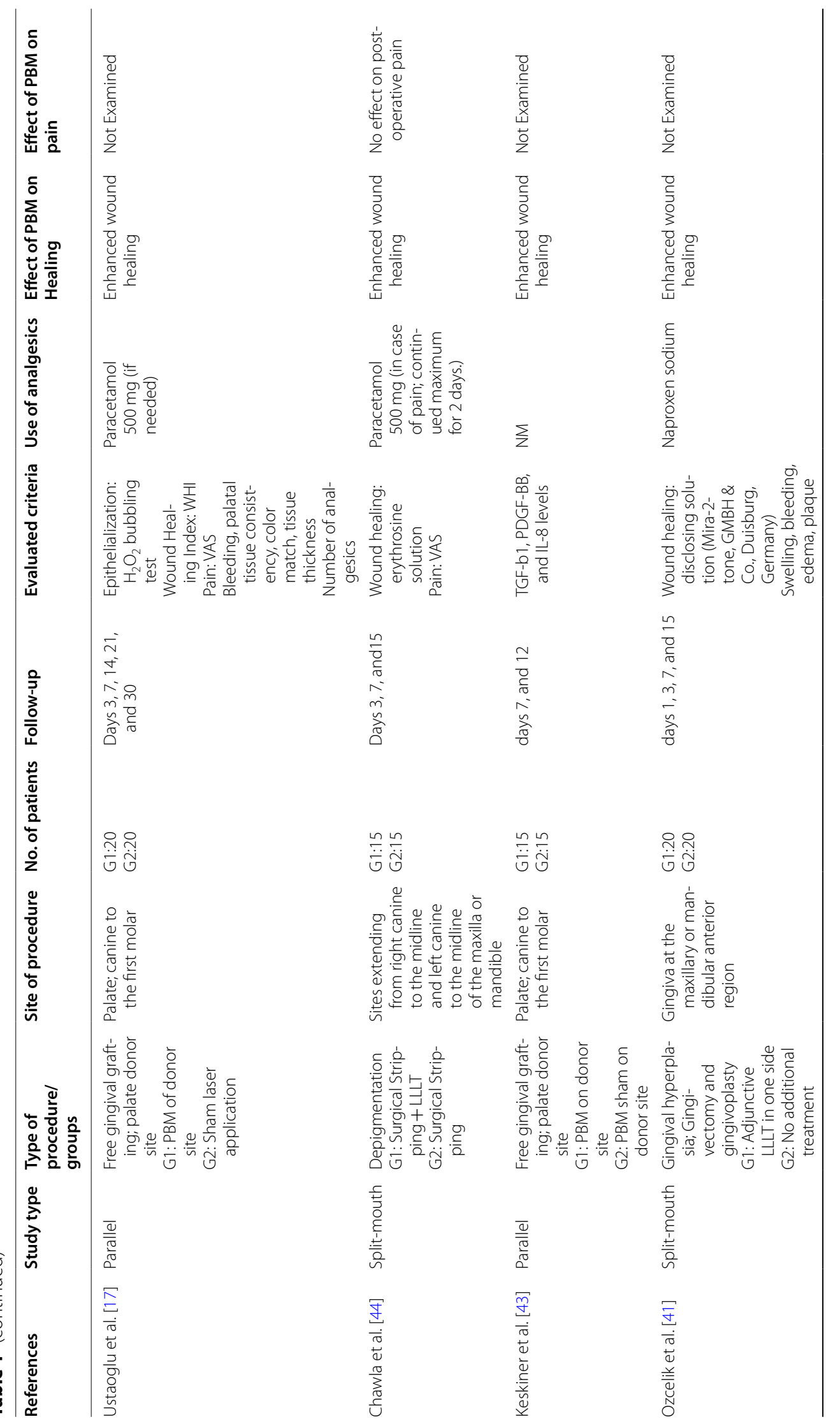




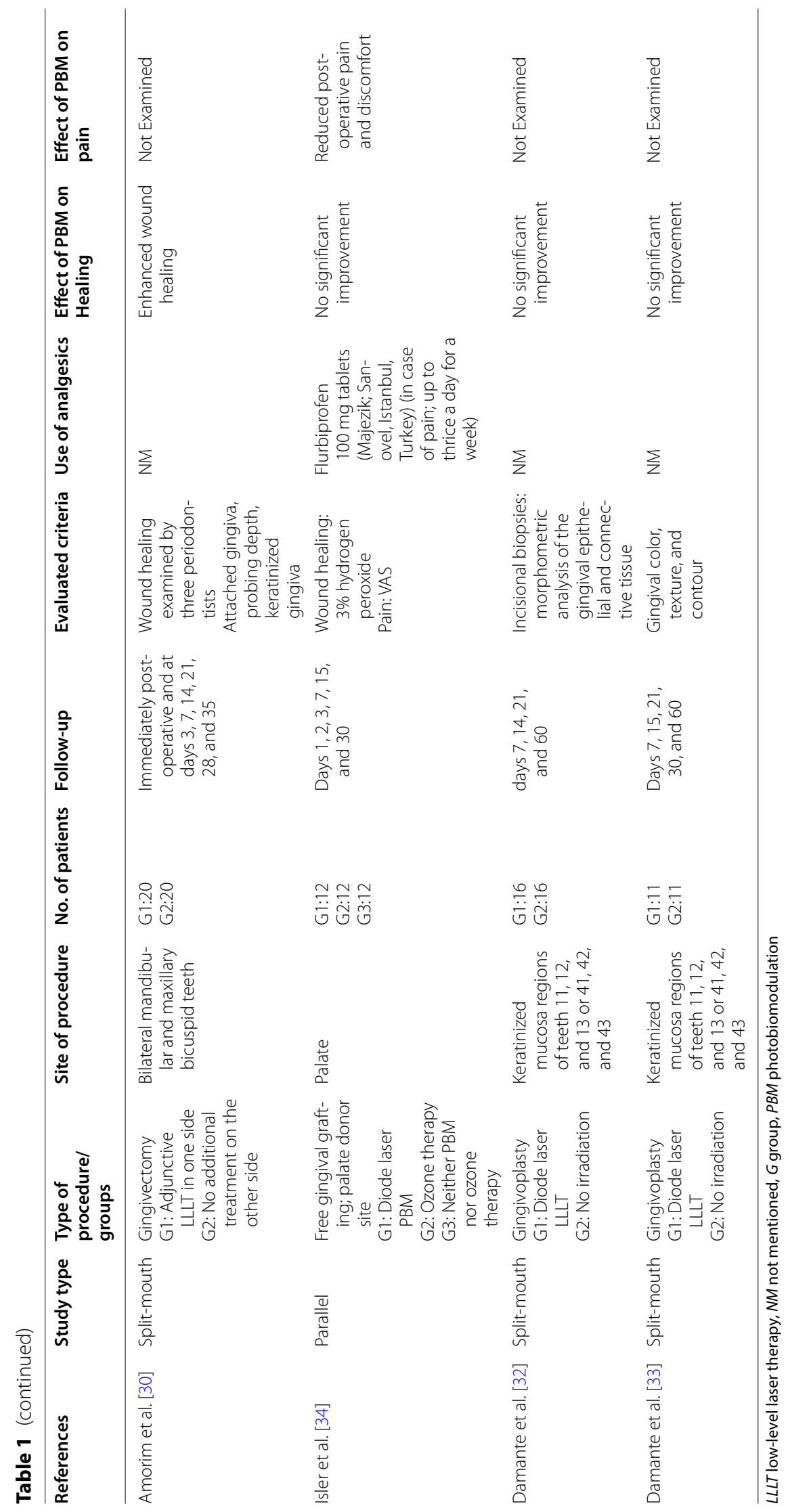




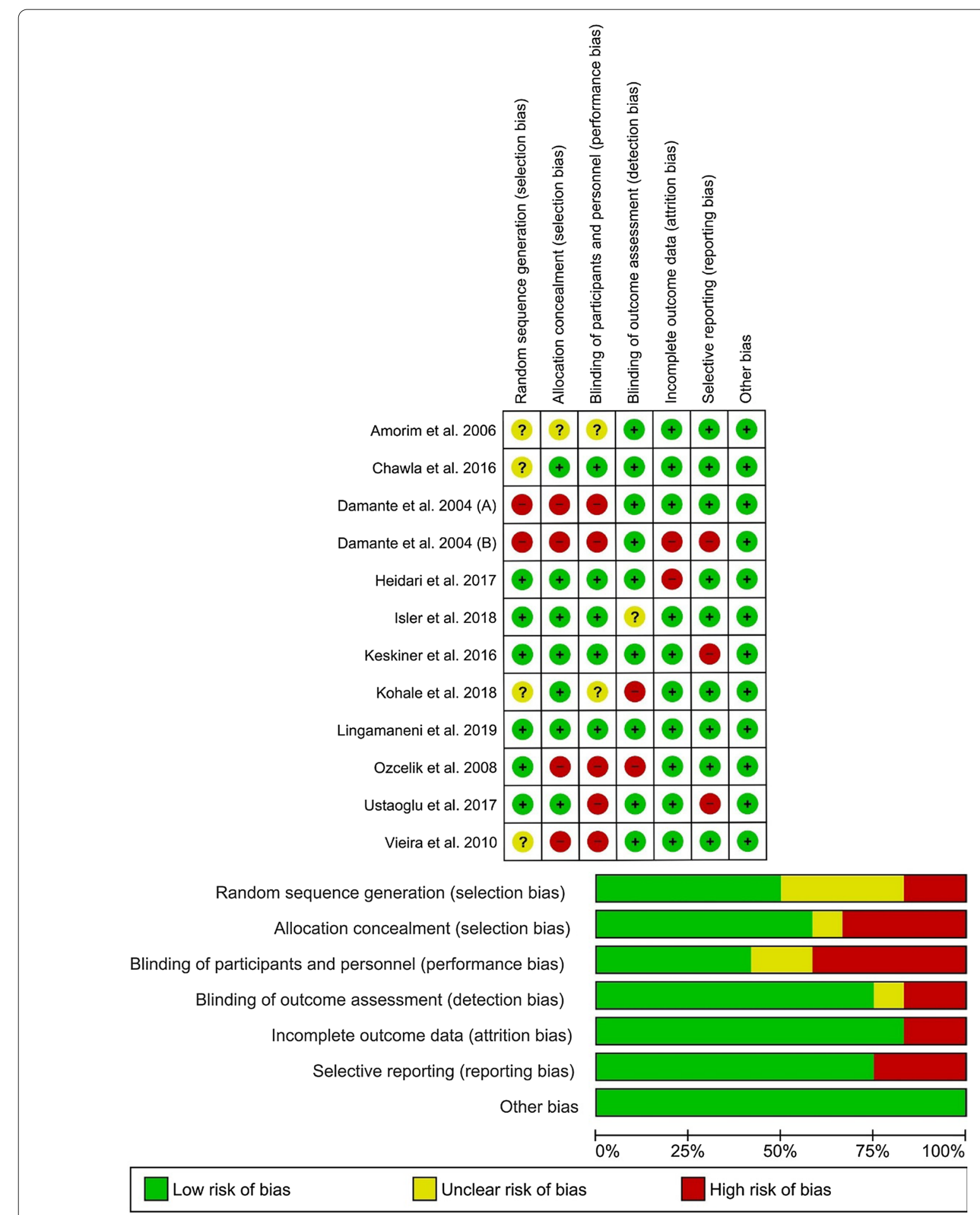

Fig. 2 Risk-of-bias analysis: a Risk of bias summary; b risk of bias graph

\section{Irradiation parameters}

Table 2 shows the characteristics of the used lasers or LEDs in the included studies. The laser types applied in 11 studies were diode [17, 19, 20, 30, 32-34, 41-44] and one study used LED [40]. The frequency of irradiation sessions was around 3-8, and a 588 to1064nm range of laser wavelengths were used. Power output and total irradiation time range were $15-5000 \mathrm{~mW}$ and 32 to $2400 \mathrm{~s}$, 


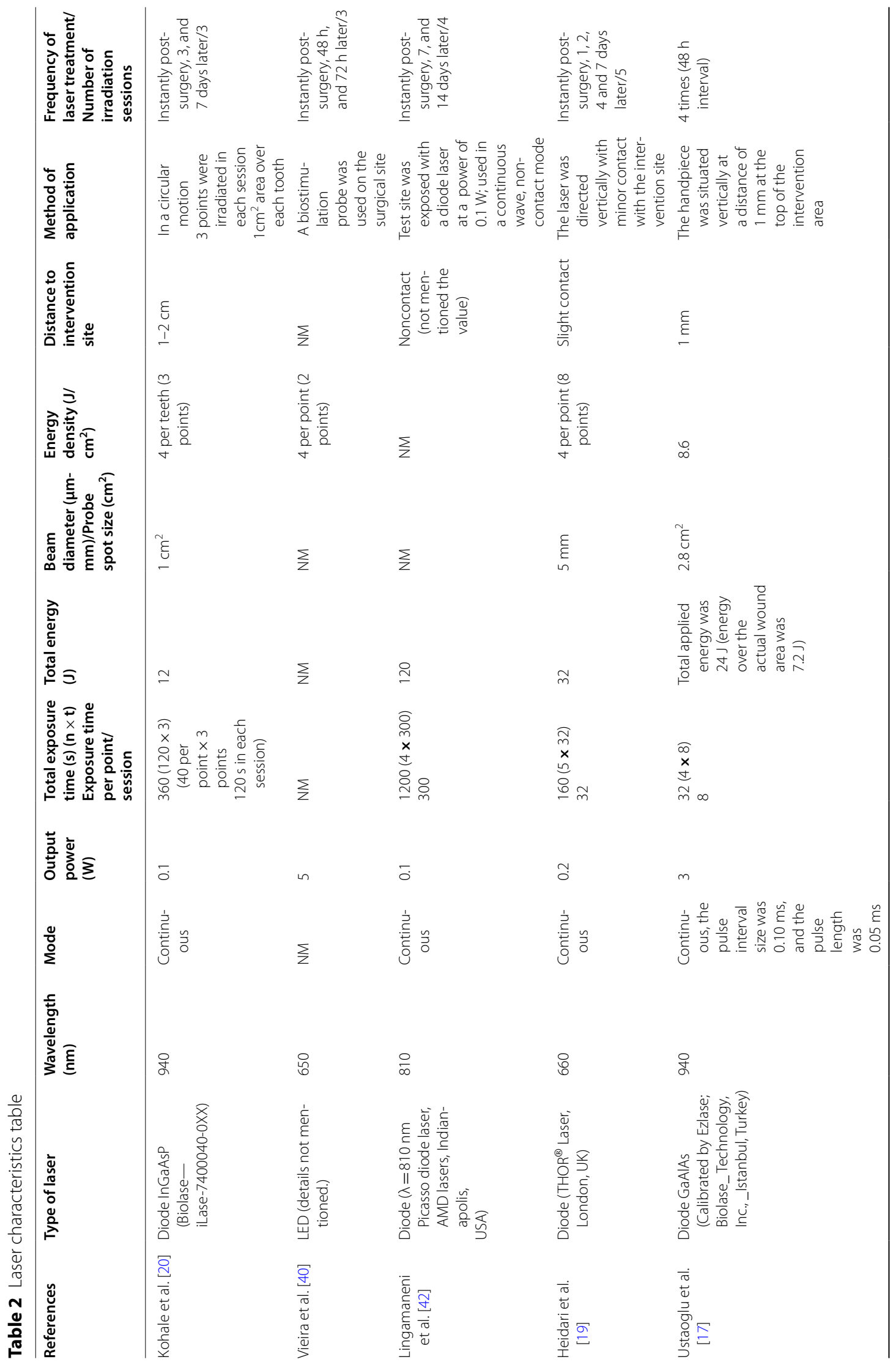




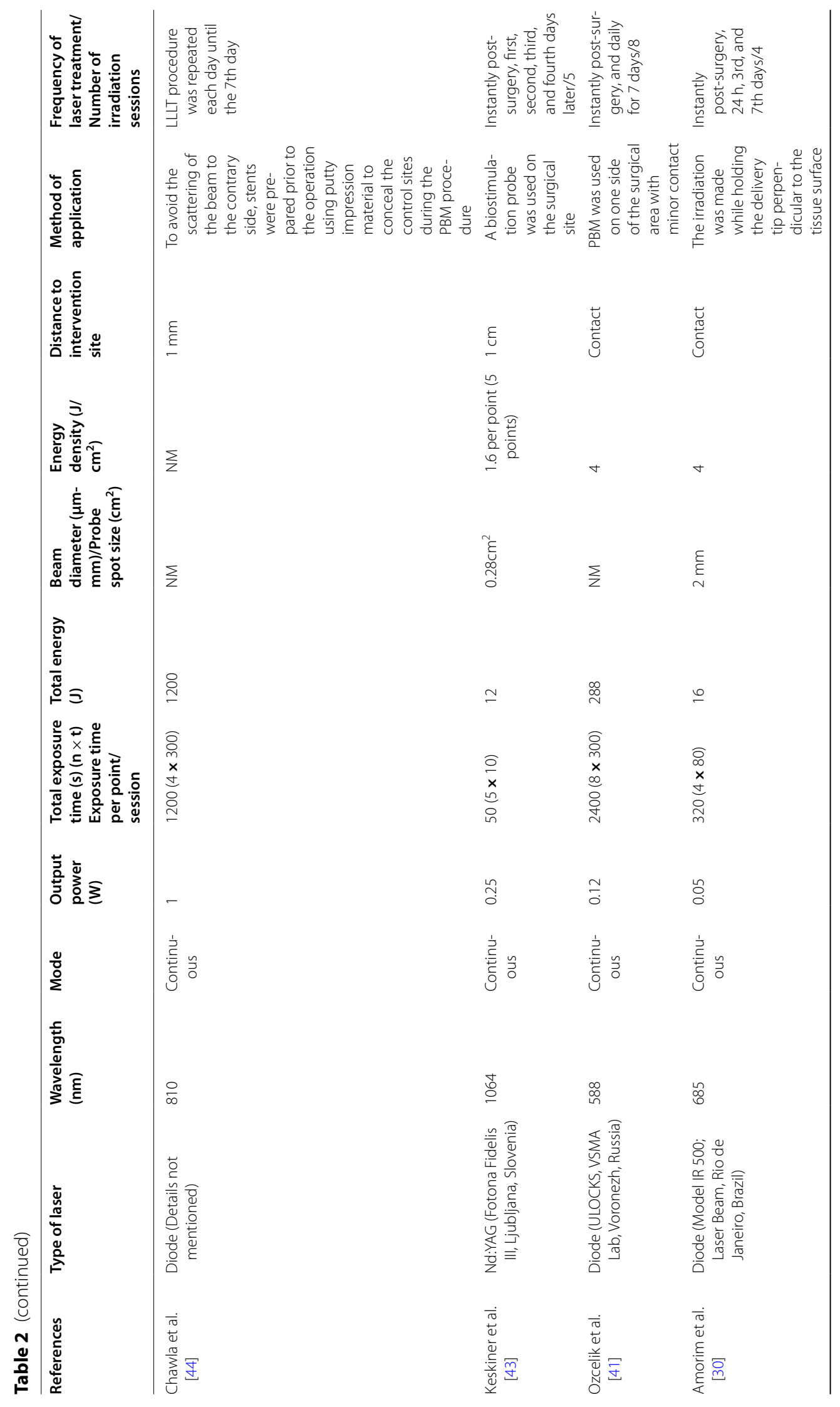




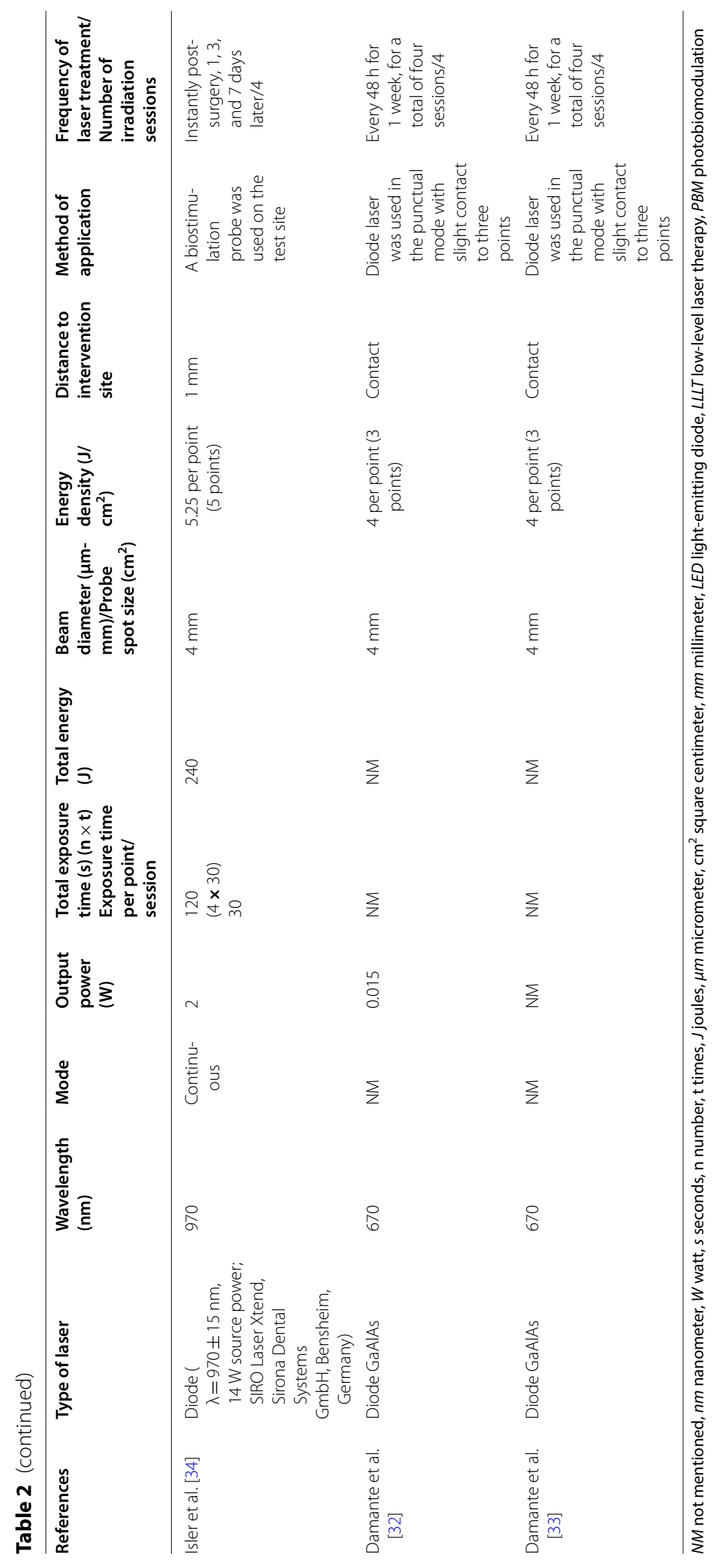


respectively. The total energy range applied to the wound area was 12-1200 J; however, some studies had not mentioned this parameter. Energy density ranged from 1.6 to $8.6 \mathrm{~J} / \mathrm{cm}^{2}$. Six studies had used the laser in a non-contact method with a distance of $1 \mathrm{~mm}$ to $2 \mathrm{~cm}$ to the surface of the tissue [17, 20,34, 42-44], and the other five studies used the laser in contact with the tissue $[19,30,32,33$, 41].

\section{Main outcomes of the studies Wound healing}

Several wound healing parameters evaluated in the included studies, included degree of epithelialization, healing index $(\mathrm{HI})$, clinical healing $(\mathrm{CH})$, Landry wound healing index (WHI), color match, tissue thickness (TT) and scar, tissue remaining wound area (RWA), tissue color and contour, and incisional biopsies for histological examinations $[17,19,20,30,32-34,40-44]$ (Table 1). Nine studies out of twelve [17, 19, 20,30,40-44] reported a significant improvement in wound healing parameters after PBM application. Damante et al. [32, 33] and Isler et al. [34] reported no stimulatory effect of PBM on wound healing. The number of included articles was not adequate to evaluate the publication bias using a funnel plot [46].

Four studies evaluated the degree of keratinization in the wound area and found that PBM can improve keratinization in secondary intention wound healing [17, 19, 20, 42]. In contrast, a study by Amorim et al. stated that there were no differences between the laser and control groups on any of the follow-ups for the amount of keratinized gingiva after gingivectomy. However, better-attached gingiva and clinical wound healing were observed, although it was not statistically significant [30]. Also, Lingamaneni et al. demonstrated that improved surface keratinization on the PBM site could not be achieved before 14th post-operative day [42].

In a study by Ozcelik, no statistically significant difference was observed between the degree of epithelialization areas in the laser and the control sites immediately after surgery. However, the intervention areas had greater epithelialization areas in comparison with the control sites at the following postoperative days [41]. Also, Vieira et al. found minor statistical significance in the PBM group for wound epithelialization after free gingival graft surgeries [40].

Ustaoglu et al. showed that tissue consistency and TT did not differ between PBM groups and controls at any time points. In contrast, the PBM group had better color matching as assessed by visual analog scale (VAS) scores compared to the control group [17].
Damante et al. (A) evaluated histologic features in wound areas that received PBM in one study and found no morphological or morphometric differences between laser and control groups. In another study by Damante et al. (B), photographs were taken for clinical evaluation. They reported that there was no advantage in using PBM to improve the wound healing outcome compared to the control group. A $670 \mathrm{~nm}$ diode laser was used in both studies [32, 33].

\section{Post-operative pain and discomfort}

The evaluated parameters regarding pain in the selected studies were mainly VAS scores and patient's pain response (NRS). Three studies showed pain relief after PBM [20, 34, 40], and two studies showed that PBM could not lead to pain relief in the wound area $[19,44]$.

Kohale et al. found that PBM can relieve pain at all evaluated time points (3, 7, 30 days) [20]. Also, Vieira et al. found that the VAS score for pain was lower in the $\mathrm{PBM}$ group from the first day to seventh, after free gingival graft surgery [40]. Isler et al. stated that although the control group had higher VAS scores at all time points, no significant differences were seen between the laser and control groups. The amount of systemic analgesic consumption did not vary between two groups. Also, patient discomfort was higher in the control group than the laser group on post-operative days [34]. In contrast, Heidary et al. found that during the first three hours post-surgery, the mean rate of VAS in the donor site was greater in the laser group in comparison with the control group. However, at longer evaluation time points, the groups did not show a substantial difference. Also, there was no difference in post-operative NSAIDs consumption between the groups [19]. Chawla et al. found that PBM cannot relieve post-operative pain in depigmentation procedures [44]. Another study by Ustaoglu et al. showed that the post-operative discomfort and the amount of analgesics did not vary through the 1st week post-surgery [17].

\section{Meta-analysis}

Two studies were eligible to participate in the metaanalysis of the Landry wound healing index $[20,42]$. The results of the analysis showed a statistically significant difference $(\mathrm{p}<0.01)$ between PBM and control groups $(\mathrm{SMD}=1.044$ [95\% CI 0.62-1.46]; $\mathrm{p}<0.01)$ in the Landry wound healing index in the 7 th post-operative day. The meta-analysis showed a large effect size and low heterogeneity $\left(I^{2}=28.9 \%\right)$ in favor of the positive effect of PBM on post-operative wound healing 7 days after surgery (Fig. 3), so the fixed effects model was used. As a publication bias test, Egger's test was not appropriate in our meta-analysis because of the insufficient number of studies included $(<10)$ [46]. 
Also, four studies were eligible to participate in the meta-analysis of complete wound epithelialization [17, $19,34,40]$. The results exhibited a statistically significant $(\mathrm{p}<0.01)$ enhancement of epithelialization in the PBM group in comparison to the control group $(R R=3.23$ [95\% CI 1.66-6.31]; $\mathrm{p}<0.01$ ) on the 14 th post-operative day. The result of the meta-analysis showed that when PBM was used, the odds of complete epithelialization was 3.2 times greater than without it. Also, there was almost no heterogeneity in the studies $\left(I^{2}<0.001 \%\right)$ favoring these results (Fig. 4). Random effects model was used in both analyses. As a publication bias test, Egger's test was not appropriate in our meta-analysis because of the insufficient number of studies included $(<10)$ [46].

\section{Discussion}

According to our search in the databases, this is the first systematic review and meta-analysis conducted to evaluate the effect of adjunctive use of PBM in periodontal surgical procedures leaving a secondary intention healing wound, such as gingivectomy, harvesting grafts from donor sites or depigmentation procedures, to assess its effectiveness on gingival healing and pain relief. All of the included studies were clinical trials, and the included non-randomized trials satisfied the pre-quality assessment.

Despite the clinical success of photobiomodulation, there are various, even contradictory theories about the actual mechanisms leading to improved clinical outcomes. The most popular and classic idea which has been challenged recently [21], is the absorption of redto-near-infrared (R-NIR) photons by cytochrome c oxidase (COX) chromophores in cellule's mitochondria that does a pivotal part in the photon-cellule interaction. The absorption process stimulates the electrons in chromophores, creating a proton gradient and ultimately leading to an increase in ATP production and glycolysis leading to higher cellular proliferation and differentiation [22]. Several studies have indicated that PBM can facilitate the speed and quality of wound healing and different mechanisms have been investigated. Keskiner et al. reported an increase in palatal wound fluid (PWF), transforming growth factor-b1 (TGF-b1), platelet-derived growth factor-BB (PDGF-BB), and interleukin-8 (IL-8) levels. This might indicate an increased rate of wound healing by stimulation of the secretion of selected mediators [43]. Enhanced collagen production, increased levels of growth factors and extracellular matrix-remodeling proteins, stimulated synthesis of adenosine triphosphate, fibroblastic proliferation, and angiogenesis, in a dosedependent manner have also been reported [16, 17, $19,20]$. It can be assumed that improved pain relief, re-epithelialization, and tissue thickness could be the direct impact of the improved wound healing process.

In a recent meta-analysis, it was stated that the mechanism and effect of PBM on primary or secondary wound healing might be significantly different [47]. This might be due to different healing mechanisms and cellular and molecular events between secondary and primary wound healing. Secondary healing involves more granulation and collagenous tissue formation in the proliferation stage, and a higher amount of remodeling and contraction in the remodeling stage of wound healing. Moreover, secondary healing is associated with more tendency to wound infection and leaves more scar tissue in the wound site [8]. Therefore, the application of PBM may more beneficial in these patients. In this study, we have only assessed the effect of PBM on secondary intention healing gingival wounds.

Although, all of the included studies in the present review have used irradiation wavelengths in the red and near infra-red range; they showed a great variation in irradiation parameters and the method of application of the adjunctive PBM therapy, making it challenging to draw evidence-based conclusions regarding the most appropriate irradiation settings needed for improvement in healing and pain relief. The most suitable laser settings for biostimulation of healing and reduction of post-operative pain of periodontal surgical wounds have not been determined yet due to the great variation observed in irradiation parameters in the available literature. Further studies with similar designs are needed to add evidence for evidence based conclusions. Factors, such as the diameter of the fiber, can alter power density and energy output in the application of lasers. It could also change the quantity of energy that is applied during the treatment, altering the wound-healing effect of PBM.

The included studies utilized various wavelengths and irradiation parameters for PBM of the wounds. Only one study used an LED $650 \mathrm{~nm}$ device reporting favourable effects on both wound healing and post-operative pain, and in one study PBM was performed using an Nd:YAG laser $(1084 \mathrm{~nm})$ device which had positive effects on healing. The other included studies used diode lasers with red to near infra-red wavelengths (588-970 $\mathrm{nm}$ ) for irradiation of the surgical sites. Energy densities ranged from 1.6 to $8.6 \mathrm{~J} / \mathrm{cm}^{2}$. The majority of studies applied an energy density of $4 \mathrm{~J} / \mathrm{cm}^{2}$ per point. However, the output powers ranged from as low as 0.05-5 W.

In the present study, despite all methodological variations, the results of the meta-analysis of the Landry wound healing index and complete wound epithelialization demonstrated a statistically significant improvement in secondary wound healing after periodontal surgeries. One of the studies from which the meta-analysis of the Landry wound 


\begin{tabular}{|c|c|c|c|c|c|c|c|}
\hline \multirow[t]{3}{*}{ Study name } & \multicolumn{7}{|c|}{ Statistics for each study } \\
\hline & \multirow{2}{*}{\multicolumn{2}{|c|}{$\begin{array}{l}\text { Std diff Standard } \\
\text { in means error }\end{array}$}} & \multicolumn{3}{|c|}{ Lower Upper } & \multirow{2}{*}{\multicolumn{2}{|c|}{ Z-Value p-Value }} \\
\hline & & & Variance & limit & limit & & \\
\hline Lingamaneni et al. 2019 & 0.566 & 0.456 & 0.208 & -0.328 & 1.460 & 1.241 & 0.215 \\
\hline \multirow[t]{2}{*}{ Kohale et al. 2018} & 1.179 & 0.242 & 0.059 & 0.704 & 1.653 & 4.865 & 0.000 \\
\hline & 1.044 & 0.214 & 0.046 & 0.624 & 1.463 & 4.879 & 0.000 \\
\hline
\end{tabular}

Fig. 3 Forest plots of Landry wound healing index in the 7th postoperative day
Std diff in means and $95 \%$ CI

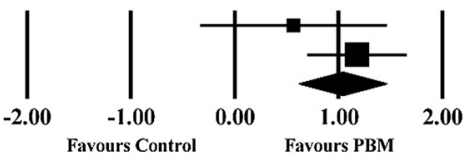

Study name

Risk Lower Upper

ratio limit limit

$\begin{array}{llll}\text { Isler et al. } 2018 \quad 7.000 & 0.400 & 122.442\end{array}$

Heydari et al. $2017 \quad 7.000 \quad 0.400 \quad 122.442$

Ustaoglu et al. $2017 \quad 3.706$

Vieira et al. 2010

2.000

3.237 $\begin{array}{lr}1.519 & 9.041\end{array}$

0.627

1.661

6.377
6.310

1.333
1.333
2.879
1.172
3.450

0.183
0.183
0.004
0.241
0.001

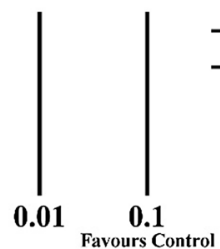

Risk ratio and $95 \%$ CI

Fig. 4 Forest plots of complete wound epithelialization in the 14th postoperative day

healing index was conducted, had a very high quality with no risk of bias [42]. The other study did not blind the outcome assessor and had detection bias with lower quality [20]. Four studies were used for the meta-analysis of the complete wound epithelialization and only one of them had high quality with low risk of bias [34]. One had incomplete outcome data (attrition bias) [19], one study had not blinded the participants or personnel (performance bias) and also had some selective reporting (reporting bias) [17], and one had issues with randomization process (selection bias) and blinding of the participants or personnel (performance bias) with relatively low quality [40].

Based on the included studies, it appears that PBM can be beneficial in improving secondary wound healing after certain types of periodontal surgeries. However, the included studies showed some controversies about the efficiency of PBM on post-operative pain. These results may be due to two factors: Firstly, pain measurement is subjective. Secondly, although the VAS scale is a valid method, the range of results is widely heterogeneous [48]. Moreover, the method of pain sensation evaluation varied in the studies. For example, some of the studies used external stimuli to measure pain. However, a recent systematic review on photobiomodulation and acute pain has indicated positive results for PBM and reported similar effects to NSAIDs consumption [49].

The summary of the risk of bias evaluation is shown in Fig. 2. The main source of bias in the included studies was the performance bias, which shows that most of the studies did not focus on blinding the participants and personnel.
To reduce this type of bias, the researchers could use sham lasers in the control sites. Or they could use the same laser in the control sites without pressing the button just to mimic the application of PBM. Moreover, to blind the operator; a person not involved in the study design could be asked to activate the laser in the specified sites.

Another main source of bias was the selection bias. Random sequence and concealment of allocation are considered of great importance in any study. Future studies should pay more attention to these risks in their studies.

\section{Study limitations}

In the present study, we did not include studies in which the patients had specific risk factors such as smoking or diabetes as there was not enough data on possible systemic complications and PBM therapy. Also, due to incomplete information and methodological heterogeneity, variable laser parameters, and methods of its application, the authors couldn't perform a meta-analysis for all of the variables in the included studies. Regarding post-operative pain and discomfort, considerable heterogeneity existed among the evaluation methods. For instance, in some studies, VAS was evaluated by application of an external stimuli like air spray $[48,50]$, while in other studies no stimulation method was utilized. One of the included studies used the NRS index to evaluate post-operative pain [20]. Overall, because of the different methods used to evaluate post-operative pain in these studies, the criteria for a meta-analysis were not met.

Furthermore, when assessing the tissue epithelialization, the existing diversity in the used methods like the 
evaluation of pictures taken from intervention areas [30], visual inspection of the wound [17, 20,34,40,41], or the use of computer software [44] did not allow us to perform a meta-analysis. The exact area of the initial wounds was not mentioned in any of the studies, which may be an interesting factor to consider in future study designs evaluating the effect of PBM in wound healing.

\section{Conclusion}

Based on the results of the current systematic review, it may be suggested that the application of PBM is a beneficial adjunct to promote second intention wound healing in periodontal soft tissue surgeries.

Currently, no optimal laser application settings can be suggested due to the extensive heterogeneity of laser parameters and variable study designs. Studies with a low risk of bias, especially in randomization and blinding, are needed to produce high-quality evidence. Also, further studies using comparable irradiation criteria with larger sample sizes and longer follow-ups on a similar procedure are necessary to indicate which parameters have essential roles in using PBM to accelerate the secondary intention healing in gingival wounds.

\section{Abbreviations}

PBM: Photobiomodulation; LLLT: Low-level laser therapy; LED: Light emitting diode; CAL: Clinical attachment level; PD: Probing depth; WHI: Wound healing Index; RR: Relative risk; MD: Mean differences; HI: Healing index; $\mathrm{CH}$ : Clinical healing; TT: Tissue thickness; RWA: Remaining wound area; VAS: Visual analog scale; NSAIDs: Nonsteroidal anti-inflammatory drugs; PWF: Palatal wound fluid; TGF-b1: Transforming growth factor-b1; PDGF-BB: Platelet-derived growth factor-BB; IL-8: Interleukin-8; R-NIR: Red-to-near-infrared; COX: Cytochrome C Oxidase; ATP: Adenosine triphosphate.

\section{Supplementary Information}

The online version contains supplementary material available at https://doi. org/10.1186/s12903-021-01611-2.

Additional file 1. Search strategies of the study.

Additional file 2. Excluded full-text titles with related reasons.

\section{Acknowledgements}

The research protocol was approved \& Supported by the Student Research Committee, Tabriz University of Medical Sciences (grant number: 64633).

\section{Authors' contributions}

$\mathrm{NV}$ and $\mathrm{MH}$ independently conducted the database searching process and the screening of the articles was done by AD, FN and LG. LN and RT did the risk of bias assessment of the studies. MH performed the meta-analysis and PE, $\mathrm{RF}, \mathrm{MH}$ and LG participated in writing the article manuscript. All authors have read and approved the final manuscript.

\section{Funding}

Not applicable.

\section{Availability of data and materials}

All data supporting the conclusions of this article are included within the article (and its additional files).

\section{Declarations}

Ethics approval and consent to participate

Not applicable.

\section{Consent for publication}

Not applicable.

\section{Competing interests}

The authors declare that they have no competing interests.

\section{Author details}

${ }^{1}$ Student Research Committee, Faculty of Dentistry, Tabriz University of Medical Sciences, Tabriz, Iran. ${ }^{2}$ Department of Oral Biological and Medical Sciences, Faculty of Dentistry, University of British Columbia, Vancouver, Canada.

${ }^{3}$ Research Center for Evidence-Based Medicine, A Joanna Briggs Institute Affiliated Center, Tabriz University of Medical Sciences, Tabriz, Iran. ${ }^{4}$ Emergency Medicine Research Team, Tabriz University of Medical Sciences, Tabriz, Iran. ${ }^{5}$ Tabriz Health Services Management Research Center, Faculty of Management and Medical Informatics, Tabriz University of Medical Sciences, Tabriz, Iran. ${ }^{6}$ Department of Periodontology, Dental Faculty, Laser Research Center in Medical Sciences, AJA University of Medical Sciences, Tehran, Iran. ${ }^{7}$ Department of Periodontics, Dental Research Center, School of Dentistry, Hamadan University of Medical Sciences, Shahid Fahmideh Blvd, 654178-38741 Hamadan, Iran.

Received: 1 February 2021 Accepted: 5 May 2021

Published online: 13 May 2021

\section{References}

1. Rojas MA, Marini L, Pilloni A, Sahrmann P. Early wound healing outcomes after regenerative periodontal surgery with enamel matrix derivatives or guided tissue regeneration: a systematic review. BMC Oral Health. 2019;19(1):56.

2. Eli I, Schwartz-Arad D, Baht R, Ben-Tuvim H. Effect of anxiety on the experience of pain in implant insertion. Clin Oral Implants Res. 2003;14(1):115-8.

3. Kim S, Lee YJ, Lee S, Moon HS, Chung MK. Assessment of pain and anxiety following surgical placement of dental implants. Int J Oral Maxillofac Implants. 2013;28(2):531-5.

4. Fardal $\varnothing$, McCulloch CA. Impact of anxiety on pain perception associated with periodontal and implant surgery in a private practice. J Periodontol. 2012;83(9):1079-85.

5. Griffin TJ, Cheung WS, Zavras Al, Damoulis PD. Postoperative complications following gingival augmentation procedures. J Periodontol. 2006;77(12):2070-9.

6. Weisensee W, Scheer M, Müller L, Rothamel D, Kistler F, Bayer G, et al. Impact of anxiety parameters on prospective and experienced pain intensity in implant surgery. Implant Dent. 2012;21(6):502-6.

7. Mei CC, Lee FY, Yeh HC. Assessment of pain perception following periodontal and implant surgeries. J Clin Periodontol. 2016;43(12):1151-9.

8. Hupp JR, Tucker MR, Ellis E. Contemporary oral and maxillofacial surgery E-book. Wound Repair. Elsevier Health Sciences, 2018: 44-55.

9. Gkatzonis AM, Vassilopoulos SI, Karoussis IK, Kaminari A, Madianos PN, Vrotsos IA. A randomized controlled clinical trial on the effectiveness of three different mouthrinses (chlorhexidine with or without alcohol and C31G), adjunct to periodontal surgery, in early wound healing. Clin Oral Investig. 2018;22(7):2581-91.

10. Laugisch $O$, Ramseier CA, Salvi GE, Hagi TT, Burgin W, Eick S, et al. Effects of two different post-surgical protocols including either $0.05 \%$ chlorhexidine herbal extract or $0.1 \%$ chlorhexidine on post-surgical plaque control, early wound healing and patient acceptance following standard periodontal surgery and implant placement. Clin Oral Investig. 2016;20(8):2175-83.

11. Lee J, Park JC, Jung UW, Choi SH, Cho KS, Park YK, et al. Improvement in periodontal healing after periodontal surgery supported by nutritional supplement drinks. J Periodontal Implant Sci. 2014;44(3):109-17.

12. Dastoor SF, Travan S, Neiva RF, Rayburn LA, Giannobile WV, Wang HL. Effect of adjunctive systemic azithromycin with periodontal surgery in the 
treatment of chronic periodontitis in smokers: a pilot study. J Periodontol. 2007;78(10):1887-96.

13. Bashutski JD, Eber RM, Kinney JS, Benavides E, Maitra S, Braun TM, et al. The impact of vitamin $D$ status on periodontal surgery outcomes. J Dent Res. 2011;90(8):1007-12.

14. Westfelt E, Nyman S, Socransky S, Lindhe J. Significance of frequency of professional tooth cleaning for healing following periodontal surgery. J Clin Periodontol. 1983;10(2):148-56.

15. Pulikkotil SJ, Nath S. Fibrin sealant as an alternative for sutures in periodontal surgery. J Coll Phys Surg Pak. 2013;23(2):164-5.

16. Yildiz MS, Gunpinar S. Free gingival graft adjunct with low-level laser therapy: a randomized placebo-controlled parallel group study. Clin Oral Investig. 2019;23(4):1845-54.

17. Ustaoglu G, Ercan E, Tunali M. Low-level laser therapy in enhancing wound healing and preserving tissue thickness at free gingival graft donor sites: a randomized. Controlled Clinical Study Photomed Laser Surg. 2017;35(4):223-30

18. Hamblin MR. Mechanisms and applications of the anti-inflammatory effects of photobiomodulation. AIMS Biophys. 2017:4(3):337-61.

19. Heidari M, Paknejad M, Jamali R, Nokhbatolfoghahaei H, Fekrazad R, Moslemi N. Effect of laser photobiomodulation on wound healing and postoperative pain following free gingival graft: a split-mouth triple-blind randomized controlled clinical trial. J Photochem Photobiol B. 2017;172:109-14.

20. Kohale BR, Agrawal AA, Raut CP. Effect of low-level laser therapy on wound healing and patients' response after scalpel gingivectomy: a randomized clinical split-mouth study. J Ind Soc Periodontol. 2018;22(5):419.

21. Sommer AP, Schemmer P, Pavláth AE, Försterling H-D, Mester ÁR, Trelles MA. Quantum biology in low level light therapy: death of a dogma. Ann Transl Med. 2020;8(7):440.

22. Dompe C, Moncrieff L, Matys J, Grzech-Leśniak K, Kocherova I, Bryja A, et al. Photobiomodulation-underlying mechanism and clinical applications. J Clin Med. 2020;9(6):56.

23. Khan I, Arany P. Biophysical approaches for oral wound healing: emphasis on photobiomodulation. Adv Wound Care. 2015;4(12):724-37.

24. Karu TI. Cellular and molecular mechanisms of photobiomodulation (lowpower laser therapy). IEEE J Sel Top Quantum Electron. 2014;20(2):143-8.

25. Doshi $\mathrm{S}$, Jain $\mathrm{S}$, Hegde R. Effect of low-level laser therapy in reducing dentinal hypersensitivity and pain following periodontal flap surgery. Photomed Laser Surg. 2014;32(12):700-6.

26. Heidari M, Fekrazad R, Sobouti F, Moharrami M, Azizi S, Nokhbatolfoghahaei $\mathrm{H}$, et al. Evaluating the effect of photobiomodulation with a 940-nm diode laser on post-operative pain in periodontal flap surgery. Lasers Med Sci. 2018;33(8):1639-45.

27. Etemadi A, Sadeghi M, Dadjou MH. The effects of low level 660nm laser irradiation on pain and teeth hypersensitivity after periodontal surgery. $\rfloor$ Lasers Med Sci. 2011:2(3):103-8

28. Yan J, Zhang J, Zhang Q, Zhang X, Ji K. Effectiveness of laser adjunctive therapy for surgical treatment of gingival recession with flap graft techniques: a systematic review and meta-analysis. Lasers Med Sci. 2018;33(4):899-908.

29. Kreisler M, Al Haj H, d'Hoedt B. Clinical efficacy of semiconductor laser application as an adjunct to conventional scaling and root planing. Lasers Surg Med. 2005;37(5):350-5.

30. Amorim JCF, De Sousa GR, Silveira LDB, Prates RA, Pinotti M, Ribeiro MS. Clinical study of the gingiva healing after gingivectomy and low-level laser therapy. Photomed Laser Surg. 2006;24(5):588-94.

31. Beresescu G, Monea M, Porca B, Cocan A, Monea A. Effects of low level laser therapy on bone regeneration of intrabony defects. In: Iulian A, Marian M, Cosmin S, Madalina A, editors.: Trans Tech Publications Ltd; 2014. p. 151-4.

32. Damante CA, Greghi SL, Sant'Ana AC, Passanezi E, Taga R. Histomorphometric study of the healing of human oral mucosa after gingivoplasty and low-level laser therapy. Lasers Surg Med. 2004;35(5):377-84.

33. Damante CA, Greghi SL, Sant'ana AC, Passanezi E. Clinical evaluation of the effects of low-intensity laser (GaAlAs) on wound healing after gingivoplasty in humans. J Appl Oral Sci. 2004;12(2):133-6.
34. Isler SC, Uraz A, Guler B, Ozdemir Y, Cula S, Cetiner D. Effects of laser photobiomodulation and ozone therapy on palatal epithelial wound healing and patient morbidity. Photomed Laser Surg. 2018;36(11):571-80.

35. Moslemi N, Heidari M, Fekrazad R, Nokhbatolfoghahaie H, Yaghobee S, Shamshiri A, et al. Effect of $660 \mathrm{~nm}$ low power laser on pain and healing in palatal donor sites a randomized controlled clinical trial. J Oral Med. 2014;27(1):71-7.

36. Moher D, Liberati A, Tetzlaff J, Altman DG, Group P. Preferred reporting items for systematic reviews and meta-analyses: the PRISMA statement. PLoS Med. 2009;6(7):e1000097.

37. HigginsJPT G. Cochrane handbook for systematic reviews of interventions version 5.1. 0 [updated March 2011]. The cochrane collaboration. 2011:20011.

38. Higgins JPT, Thomas J, Chandler J, Cumpston M, Li T, Page MJ, Welch VA (eds). Cochrane Handbook for Systematic Reviews of Interventions version 6.1 (updated September 2020). Cochrane, 2020. www.training.cochrane. org/handbook.

39. Egger M, Smith GD, Schneider M, Minder C. Bias in meta-analysis detected by a simple, graphical test. BMJ. 1997;315(7109):629-34.

40. Vieira J, Lopes CB, DeMarco AC, de Melo Filho AB, Jardini MAN. Clinical study of laser biomodulation (650 $\lambda$ ) after free gingival grafts. J Oral Laser Appl. 2010;10:159-63.

41. Ozcelik O, Cenk Haytac M, Kunin A, Seydaoglu G. Improved wound healing by low-level laser irradiation after gingivectomy operations: a controlled clinical pilot study. J Clin Periodontol. 2008;35(3):250-4

42. Lingamaneni S, Mandadi LR, Pathakota KR. Assessment of healing following low-level laser irradiation after gingivectomy operations using a novel soft tissue healing index: a randomized, double-blind, split-mouth clinical pilot study. J Indian Soc Periodontol. 2019;23(1):53.

43. Keskiner I, Lutfioğlu M, Aydogdu A, Saygun NI, Serdar MA. Effect of photobiomodulation on transforming growth factor- $\beta 1$, platelet-derived growth factor-BB, and interleukin-8 release in palatal wounds after free gingival graft harvesting: a randomized clinical study. Photomed Laser Surg. 2016;34(6):263-71.

44. Chawla K, Lamba AK, Tandon S, Faraz F, Gaba V. Effect of low-level laser therapy on wound healing after depigmentation procedure: a clinical study. J Indian Soc Periodontol. 2016;20(2):184.

45. Sobouti F, Khatami M, Heydari M, Barati M. The role of low-level laser in periodontal surgeries. J Lasers Med Sci. 2015;6(2):45-50.

46. Jin Z-C, Wu C, Zhou X-H, He J. A modified regression method to test publication bias in meta-analyses with binary outcomes. BMC Med Res Methodol. 2014;14(1):132

47. Zhao H, Hu J, Zhao L. The effect of low-level laser therapy as an adjunct to periodontal surgery in the management of postoperative pain and wound healing: a systematic review and meta-analysis. Lasers Med Sci. 2020:56:1-13.

48. Dias SBF, Fonseca MVA, dos Santos NCC, Mathias IF, Martinho FC, Junior MS, et al. Effect of GaAlAs low-level laser therapy on the healing of human palate mucosa after connective tissue graft harvesting: randomized clinical trial. Lasers Med Sci. 2015;30(6):1695-702.

49. Bjordal JM, Johnson MI, Iversen V, Aimbire F, Lopes-Martins RAB. Low-level laser therapy in acute pain: a systematic review of possible mechanisms of action and clinical effects in randomized placebo-controlled trials. Photomed Laser Surg. 2006;24(2):158-68.

50. da Silva Neves FL, Silveira CA, Dias SB, Santamaria Júnior M, de Marco AC, Kerbauy WD, et al. Comparison of two power densities on the healing of palatal wounds after connective tissue graft removal: randomized clinical trial. Lasers Med Sci. 2016;31(7):1371-8.

\section{Publisher's Note}

Springer Nature remains neutral with regard to jurisdictional claims in published maps and institutional affiliations. 